pityriasis rosea, tinea corporis, nummular dermatitis and prurigo nodularis. Guttate psoriasis can spontaneously fade within several weeks or several months, phototherapy as a first-line treatment has a good effect, and antibiotics may be used if persisting infection is suspected. Overall, most patients have a good prognosis, just a few patients have a chronic course.

\author{
Rouyu Fang and Qiuning Sun* \\ Department of Dermatology \\ Peking Union Medical College Hospital, \\ Chinese Academy and Medical Sciences, \\ Peking Union Medical College, \\ Beijing,China. \\ *doctorjenny1@126.com
}

\title{
Traumatic Anserine Folliculosis
}

A 10-year-old boy presented with asymptomatic roughness over the left cheek since 6 months. He acknowledged resting in a particular position, which led to prolonged localized pressure and friction, while watching television or studying. Examination revealed multiple tiny skin-coloured, discrete but grouped, follicular papules having a sandpaper-like feel (Fig. 1). Considering the site of affection and characteristic history, a diagnosis of traumatic anserine folliculosis was established. He was treated with topical tretinoin cream, and advised to avoid trauma and friction to the area.

Traumatic anserine folliculosis is an under-recognized condition characterized by multiple, closely set grouped follicular papules affecting the chin, jaws, and neck. This entity should be differentiated from keratosis pilaris (keratinous follicular plugs, usually surrounded by erythema), lichen spinulosus (pruritic symmetric plaques having thorny grouped follicular papules), trichostasis spinulosa (hair tufts through follicle, resembling comedones), and trichodysplasia spinulosa (viral infection

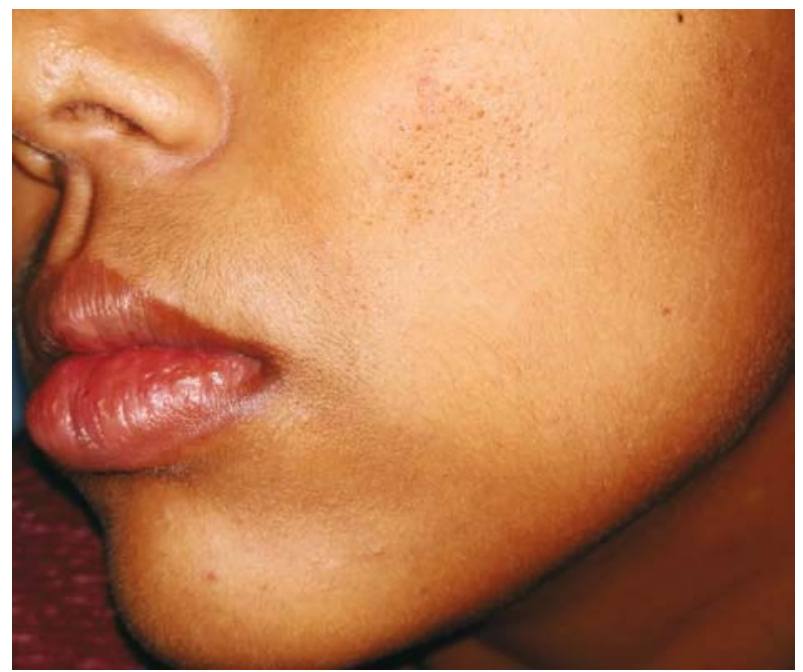

FIG. 1 Skin-colored, discrete but grouped, follicular papules over left cheek.

in immunocompromised). Treatment includes topical keratolytics and removal of etiological factor.

Abheek Sil* and Anupam Das

Department of Dermatology, Venereology, and Leprosy, $R G$ Kar Medical College, Kolkata, West Bengal, India.

*abheek.sil@gmail.com

\section{Becker Melanosis}

A 12-year-old boy presented with a gradually progressive asymptomatic area of discoloration over right forearm since last 2 years. Examination revealed a unilateral, well-circumscribed $6 \mathrm{~cm} \times 8 \mathrm{~cm}$ tan-brown patch on the right forearm, and having irregular border and blotchy pigmentation at the periphery (Fig. 1). Localized coarse hair and acneiform eruptions were observed, restricted to the patch. Darier sign was negative. No skeletal, soft tissue or neurological abnormalities were

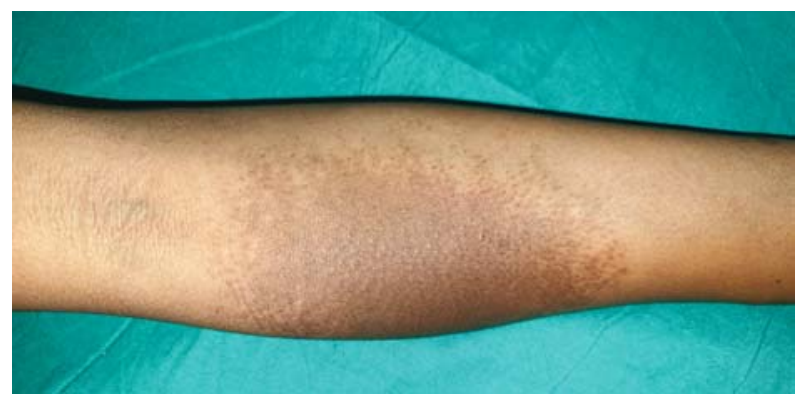

Fig. 1 Well-circumscribed tan-brown patch on the forearm, having irregular border and blotchy pigmentation at the periphery with localized coarse hair and acneiform eruption. 